\title{
Philosophiques
}

\section{Serge Robert, Les mécanismes de la découverte scientifique, Ottawa, Les presses de l'Université d'Ottawa, 1993.}

\section{Don Ross}

Volume 23, numéro 1, printemps 1996

Critères esthétiques et métamorphoses du beau

URI : https://id.erudit.org/iderudit/027390ar

DOI : https://doi.org/10.7202/027390ar

Aller au sommaire du numéro

Éditeur(s)

Société de philosophie du Québec

ISSN

0316-2923 (imprimé)

1492-1391 (numérique)

Découvrir la revue

Citer ce compte rendu

Ross, D. (1996). Compte rendu de [Serge Robert, Les mécanismes de la découverte scientifique, Ottawa, Les presses de l'Université d'Ottawa, 1993.]

Philosophiques, 23(1), 204-210. https://doi.org/10.7202/027390ar d'utilisation que vous pouvez consulter en ligne.

https://apropos.erudit.org/fr/usagers/politique-dutilisation/ 
Serge Robert, Les mécanismes de la découverte scientifique, Ottawa, Les presses de l'Université d'Ottawa, 1993.

J'imagine aisément que plusieurs philosophes des sciences ont dũ s'étonner devant l'étendue et l'ambition du nouveau livre de Serge Robert. J'étais du nombre à partir de la page dix, là où Robert énumère les problèmes qu'il entend résoudre, ou sur lesquels il espère, à la limite, faire des progrès. Cette liste inclut la question de la nature et de la signification épistémologique de la perception, celle du rōle des définitions en science, de la structure des théories scientifiques, des présuppositions métaphysiques de la science, la question des caractéristiques de la méthode scientifique, du critère de démarcation entre science et pseudo-science, celle de savoir s'il y a et comment s'opère le progrès scientifique, celle de la logique de la découverte scientifique, celle de savoir le type de capacités cognitives qui pourraient être requises pour les futurs scientifiques. On voit immédiatement qu'à toute fin pratique, cette liste inclut toutes les questions principales qui ont préoccupé les philosophes des sciences pendant les quatre-vingt demières années (et peut-ètre même depuis Descartes). Mais, bravement, Robert fait part de son intention, qui est de développer une théorie qui permette de cerner tous ces aspects de la science, et ceci en deux cent cinquante pages environ. S'il nous avait avertis à l'avance, comme il aurait pu le faire, que le livre contient beaucoup de descriptions de conceptions philosophiques existantes ou possibles, mais très peu en matière d'arguments, notre inquiétude ne se serait qu'intensifier. Robert peut-il, aurions-nous été tentè de demander, être sërieux? 
La rêponse est, bien sûr, qu'il l'est. Il est si clairement bien versé dans la littérature de son sujet que le caractère ambitieux de son projet a naturellement dũ lui être évident. Toutefois, je voudrais, dans les pages qui suivent, d'abord donner une interprétation du travail de Robert qui rende son objectif à la fois modeste et potentiellement éclairant. Je soutiendrai ensuite que cet objectif n'en demeure pas moins irréalisable. Cette critique se veut constructive. Elle émergera de méta-réflexions sur le type de projets qu'ont entrepris les philosophes des sciences et sur la viabilité relative de ces divers projets. Dans cette perspective. le principal point de désaccord entre Robert et moi se ramènera à ceci : je suis sceptique à l'égard de toute forme de théorie générale en philosophie des sciences, tandis qu'il est, pour sa part, manifestement confiant dans les perspectives d'avenir de ce genre de thêories. J'ose espérer que cette tentative, qui vise à identifier le nœud et la signification de notre divergence. se révélera être d'une grande utilité.

Le premier puzzle que j'eus à résoudre, en essayant de trouver la meilleure manière d'aborder la lecture du livre de Robert. fut celui de m'expliquer pourquoi il y avait si peu d'arguments en tant que tels. Comment. s'interroge le lecteur naif, Robert peut-il s'imaginer qu'une théorie compréhensive de la science puisse ètre simplement proclamée? La réponse réside, à ce qu'il me semble, dans la conception, implicite chez Robert, selon laquelle le plus important travail argumentatif aurait été déjà accompli dans l'histoire récente de la discipline. Il semble que Robert croit que de ce travail argumentatif n'ont résulté que des espèces de cul-de-sac, où il semble impossible de décider entre des visions opposées, puisque chacune met fortement à contribution nos intuitions, mais en les tirant dans des directions contraires. J'impute cette conception à Robert sur la base de la stratégie dont il se sert sans relâche dans son livre. Cette stratégie consiste à montrer, en prenant deux à deux des conceptions philosophiques opposées, que les conflits qui semblent évidents entre elles ne sont qu'apparents, puisque toutes nos intuitions peuvent être simultanément satisfaites au moyen d'une theorie qui comprend l'aspect de la pratique scientifique que chacune de ces conceptions antérieures de la science aurait correctement saisi, mais qui ne comprendrait précisement que cet aspect. Par ce moyen, Robert tente par exemple de réconcilier les duos suivants : constructivisme et empirisme, externalisme et internalisme, descriptivisme et rigidisme concernant les termes référentiels, essentialisme et nominalisme, approches sémantiques et syntaxiques de la structure des théories scientifiques, méthodes déductiviste et inductiviste, vérificationnalisme et faillibilisme. $\dot{A}$ vrai dire, cette approche a effectivement l'avantage de ne réclamer qu'un minimum d'arguments : en effet, s'il s'avérait que, dans la plupart sinon dans tous les cas, son diagnostic de l'origine de nos intuitions conflictuelles était fondé, et que sa réconciliation de doctrines opposées était cohérente, alors nous n'aurions plus besoin de réclamer une autre forme de persuasion.

Si cette description du projet de Robert est juste, alors s'offrent à nous trois voies par lesquelles nous pouvons le soumettre à des pressions critiques. Premièrement, nous pouvons nous demander si Robert est effectivement justifié de croire que l'on peut atteindre une image philosophiquement éclairante de la science grâce à la réconciliation des thèses directrices traditionnelles dans les diverses parties de l'épistémologie des sciences. Deuxièmement, nous pouvons aussi nous interroger à savoir si le nombre de réconciliations qui s'avèrent réussies est suffisant pour justifier la confiance que Robert semble mettre dans l'ensemble même de son projet. Troisièmement, on peut douter du fait que l'aggrégation de ces compromis saura former un tout suffisamment cohérent 
pour constituer l'image unifiée que Robert veut qualifier de "rationalisme interactionniste *. La question que soulève la première ligne de critique semble avoir préséance sur les autres : il y aurait peu de raisons de nous engager dans les analyses détaillées que requiert l'examen des deux autres problèmes à moins qu'elle ne reçoive une réponse affirmative. Je présume que c'est par rapport à cette première question que le philosophe typique des sciences se montrera le mieux disposé à partager le point de vue de Robert. Ainsi, en m'opposant à lui sur ce point, mon désaccord est net, et, à ce point-ci, il peut mème sembler radical.

Seulement un tout petit nombre d'affirmations dans ce livre me semblent clairement fausses. Ceci ne veut pas dire que je renonce à diverger profondément avec Robert, bien au contraire. Je soutiendrais que la plupart des affirmations qui sont à proprement parler constitutives du rationalisme interactionniste ne peuvent pas ètre interprétées de façon à ètre à la fois vraies et intéressantes, dans le sens où elles auraient quelque chose d'éclairant et de substantiel à nous dire sur la science. Je m'appuierai, pour illustrer cela, sur un exemple tiré de l'analyse que Robert fait de la logique de la perception : discussion qui est la source même de plusieurs des distinctions conceptuelles qui sont utilisées plus tard dans le livre. Tout au début de la discussion. Robert déclare que "la distinction [entre le donné et le construit] existe, mais dans une co-présence sans opposition [p. 16] ". Cette affirmation est, me semble-t-il, manifestement vraie. En effet, si cette distinction, qui est après tout une distinction entre concepts, n'avait pas existé, il n'aurait jamais été possible d'articuler le conflit qui existe entre une conception empiriste et une conception constructiviste de la science. La thèse selon laquelle les processus de transduction de l'information, d'une part, et les processus de stockage et de représentation de l'information sous forme de résumés théoriques construits. d'autre part, travaillent de concert dans la cognition, est une thèse qui n'est. pour autant que je sache, remise en cause par personne. Les problèmes philosophiques qui émergent ici ne viennent pas de ce que nous refusons d'admettre qu'il y ait interaction entre la transduction et la construction, mais bien plutôt de ce nous ne savons pas comment rendre compte de cette interaction manifeste tout en essayant de conserver, pour les besoins de l'épistémologie, des concepts qui font abstraction de cette interaction comme celui de perception.

Aucun renfort d'analyse conceptuelle $a$ priori n'aura de succès ici, et ce pour la simple raison que de tels travaux essaient de faire l'impossible, c'est-à-dire d'établir un concept utile de la perception, qui serait plus riche que celui de transduction en ce sens qu'il permettrait le traitement de l'information concernant les objets qui se subsument sous les concepts, tout en ignorant le rôle que jouent les influences de "haut-en-bas" (top-down) lors de la production des représentations de ces concepts. Un tel problème n'embarrasse aucunement les naturalistes, car nous pouvons nous contenter de dire que si le cerveau dispose. derrière les transducteurs sensoriels, de modules de traitement d'entréeseulement d'information - comme cela semble ètre le cas - alors l'activité de ces modules est ce en quoi consiste la perception. Et s'il s'avérait que nous avons été abusés par les données empiriques, bref, que de tels modules n'existent pas, alors il nous faudrait simplement reconnaître que le concept même de perception ne sera plus d'aucune utilité. Dans une telle éventualité, notre explication devra ètre en termes de transduction et d'équilibrage en retour de type-pandemonium. Or, Robert n'est pas anti-naturaliste. D'autre part, sa méthodologie, telle qu'exposée ci-dessus du moins, n'est pas naturaliste non plus. Cependant, dans la mesure où sa propre position émerge des arguments 
de philosophes qui utilisent un concept de la perception qui n'est pas dérivé de recherches empiriques, il se retrouve avec un concept philosophique qui n'est pas raffinè, et qui est probablement l'élaboration du concept populaire vague. $\dot{A}$ la lumière de ce qui précède, considérons comment nous pourrions évaluer l'affirmation suivante: "La seule conclusion alors acceptable est de soutenir que les individus et les propriètés (et les relations) sont progressivement contruits dans notre perception par une détemination progressive des uns par les autres et des autres par les uns. "(p. 22) Cette affirmation est sürement vraie - mais elle est totalement dénuée d'information. Elle est formulée dans un discours conceptuel qui se situe à un niveau d'abstraction tellement au-dessus des faits eux-mèmes, qu'il est difficile, à un tel niveau d'abstraction, de dire quoi que ce soit dont on ne puisse pas trouver une interprétation vraie. Je voudrais risquer ici une généralisation que l'on peut modestement appeler "La Première Loi de l'Enquête Conceptuelle Pure ": lorsqu'un niveau suffisant d'abstraction du discours se trouve associé, en tant que partie intégrante de la méthodologie de lecture, avec le principe de charité, alors cela conduit inévitablement à la vacuité. La méthode dont Robert se sert pour trouver un terrain d'entente entre les conceptions rivales le conduit presque toujours à un niveau d'abstraction plus élevé que le niveau auquel le conflit apparait. Il s'ensuit donc qu'il est constamment menacé de tomber sous le coup de la Première Loi.

Puisqu'il s'agit là d'une ligne critique assez sévère, je ferais mieux, me semble-t-il, de l'appuyer par un autre exemple. Robert établit par ailleurs une distinction - qui occupe une place prééminente dans sa théorie générale entre trois niveaux différents de langages cognitifs, qu'il appelle respectivement " descriptif. explicatif et justificatif ". Ces distinctions de niveaux, pourrait-on se demander, existent-elles ? Naturellement, Robert nous explique la manière de distinguer entre ces concepts; donc, aux mains de leur créateur, ces distinctions existent. Mais, pourrait-on s'interroger, correspondent-elles pour autant à des dictinctions empiriquement motivées? Il n'existe, pour autant que je sache, aucun travail dans les sciences cognitives qui puisse servir à motiver. ou qui puisse être utilement réorganisé conceptuellement par le triumvirat de Robert. Ce fait en soi ne corrompt pas automatiquement la pertinence de ces distinctions, mais il impose à leur créateur le fardeau de montrer qu'elles sont nécessaires pour pouvoir rendre compte adéquatement de certains épisodes réels de l'histoire de la science, et non pas des épisodes reconstruits philosophiquement. Or, nulle part dans son ouvrage, Robert n'a cherché à honorer cette charge. Du coup, c'est à nous qu'échoit la tâche d'imaginer la meilleure manière d'utiliser ces catégories dans les cas concrets, ce qui nous donne la liberté d'assigner, plus ou moins selon notre propre vouloir, une interprétation empirique à ces concepts. Naturellement, si nous voulons ètre charitables, nous assignerons nos interprétations de sorte que les affirmations générales de Robert (au sujet des relations entre concepts) soient vraies - ce qui, ipso facto. en fait des affirmations qui ne nous apprennent rien de nouveau sur la science telle qu'elle est pratiquée ou sur les processus cognitifs réels.

Je suis d'avis que ma critique ne troublera pas outre mesure quelqu'un qui perçoit le paysage de la philosophie des sciences de la même manière que Robert. Car après tout, pourrait-on me rétorquer, s'il se trouve que Carnap et Popper nous ont légué des descriptions de la science qui, bien que contenant des intuitions profondes et essentielles, n'en demeurent pas moins inadéquates sur certains points, alors comment pourrait-il ne pas ètre important et utile, s'il se trouve en plus que ces descriptions sont incompatibles, que l'on nous instruise sur la manière d'utiliser ces deux perspectives pour se soutenir mutuellement ; conservant par là les deux séries d'intuitions tout en éliminant, par la même 
occasion, les difficultés auxquelles nous ont confronté leurs cadres conceptuels trop étroits ? Le principe logique qui est implicite dans cette question rhétorique est valide : en revanche, l'antécédant contient, pour sa part, quelques ambiguités. Le travail de Carnap (je suis moins sûr de celui de Popper) est certes une source de brillantes intuitions sur la logique de nos concepts épistémiques traditionnels et sur le langage avec lequel nous tentons de formuler certaines de nos conclusions scientifiques les plus importantes. Mais il n'en découle pas que Carnap réussit, en fin de compte, à nous apprendre quelque chose sur la pratique scientifique qui soit à la fois vrai et éclairant, bien que telle ait pu ètre son intention. La raison en est que pour pouvoir interpréter le travail de Carnap comme un travail qui porte sur la science telle qu'elle est pratiquée, il faut tout d'abord adhérer aux hypothèses suivantes, qui sont fausses : (1) qu'il existe un ensemble de principes épistémiques distinctifs et communs à la pratique scientifique ; et (2) que l'enrégimentation du langage de la science rendra manifeste les principes épistemiques de celle-ci. Bien que Robert semble accepter ces deux hypothèses, un projet comme le sien pourrait très bien se passer de (2). (1) reste nêanmoins cruciale. Si cette hypothèse était niée, il n'y aurait plus aucune raison de penser que la résolution de disputes conceptuelles parmi des philosophes, dont les généralisations sont faites pour s'appliquer aux différentes disciplines scientifiques, puisse contribuer à faire de la lumière sur la science telle qu'elle est pratiquée dans les disciplines particulières. Pour ce faire donc, je consacrerai le reste de ma présentation aux raisons qui m'amènent à croire que (1) est fausse.

À vrai dire, il est trompeur de parler ici de mes raisons, car mon opposition à (1) s'inspire d'un thème récent de la littérature ; thème dont les principaux tenants sont Ian Hacking et Nancy Cartwright. Leur procédure d'argumentation est principalement inductive. Des études précises de diffërents épisodes, certains historiques et d'autres contemporains, de la pratique rëelle des sciences révèlent que les scientifiques sont en général extrêmement opportunistes dans leurs épistémologies ; qu'ils sont beaucoup moins préoccupés par la mise au point de théories, et beaucoup plus intéressés par la manipulation d'objets expérimentaux, que les philosophes ne l'ont imaginé. Mon favori, parmi les exemples de Cartwight. est un exemple tiré de la théorie de la radiation quantique. Lorsque des atomes dé-excitent, en émettant des photons dans un champ de radiation, on observe un élargissement de la ligne d'émission de l'atome radioactif. Ce phénomène, connu sous le nom de damping, ne soulève aucun doute ; et mieux, les physiciens s'accordent généralement pour reconnaître que l'élargissement de la largeur de la ligne est due à l'émission de photons. Néanmoins, Cartwright décrit non moins de six différentes manières non complémentaires par lesquelles les physiciens essaient d'incorporer ce phénomène dans le cadre mathématique général de la mécanique quantique. Il ne s'agit pas là d'une simple question d'équivalence formelle. Les six approches "rivalisent l'une contre l'autre, chacune offrant une loi différente pour rendre compte du même phénomène ". Un philosophe traditionnel des sciences s'imaginera súrement que, en de telles circonstances, les physiciens sont en train de se creuser les méninges afin de réduire le nombre de solutions. En effet, tant qu'ils n'y seront pas arrivés, ils n'auront pas d'explication unifiee du phénomène en question. Or, les explications unifiées (tout au moins à l'intérieur des disciplines comme l'ont affirmé des moins ambitieux que Robert) sont supposées être la base même de l'explication de la nature, et donc l'objectif ultime des sciences. Mais, en fait, les physiciens ne sont pas en train de chercher à émonder leur ensemble de solutions. Au contraire, ils exploitent activement la liberté de manœuvre que leur procure leur trop plein de richesse. Puisque chacune de ces six théories se révèle être 
meilleure que les autres dans certains contextes expérimentaux, le physicien peut donc utiliser celle qui se trouve ètre la mieux adaptée à ses circonstances particulières. En résumé, disons ceci : il n'existe pas de théorie unique du damping quantique, et la communauté des chercheurs n'y travaille pas actuellement parce qu'elle préfere ne pas en avoir qu'une seule.

La puissance de persuasion de ce type d'exemple dépend évidemment de savoir jusqu'où il peut être typique. L'espace qui m'est alloué ici ne me permet que de vous renvoyer à Cartwright, où se trouvent présentés toute une série d'exemples qui me semble être cumulativement convaincants. Je suis. à tout le moins, convaincu qu'une situation du type de celle qu'illustre le damping quantique n'est pas du tout inhabituelle en physique. Je m'attendrais à rencontrer le même type de situation en microbiologie. En revanche. de telles situations pourraient être très rares dans l'écologie évolutionniste des populations et en microéconomie, pour ne prendre que deux exemples. La raison principale d'une telle différence, suggérerai-je, réside dans le fait que la plupart des aspects conceptuels de la physique et de la microbiologie sont suffisamment contrôlés par l'interaction physique directe qui existe entre eux et les partie pertinentes du monde, tant et si bien qu'il n'est point besoin de discipliner la prolifération théorique par le recours à des principes méta-disciplinaires. De telles conditions n'existent pas en écologie évolutionniste des populations ou en microèconomie, si bien que ces sciences, en dépit du fait qu'elles n'aient pas servi d'exemplifications pour les philosophes, sont beaucoup plus proches du type de modèles de sciences que les philosophes ont généralement endossé. Faisons ressortir deux aspects de la présente discussion qui sont pertinents pour la remise en cause de l'hypothèse (1). Tout d'abord, si Cartwright a raison, alors l'importance de principes épistémiques abstraits comme guide de l'activité des sciences fondamentales, ou mème comme caractérisation légitime du travail qui s'y fait, est bien moindre que ne l'ont supposé les philosophes des sciences dont Robert s'est inspiré. Deuxièmement, le fait même que la généralisation négative de Cartwright ne puisse s'appliquer uniformément à toutes les sciences, nous rappelle un autre fait qui a èté mis en lumière par la prolifération d'études empiriques des sciences : à savoir que les diverses sciences sont aussi différentes les unes des autres que le sont les aspects respectifs du monde qu'elles étudient. Du reste, la première série de différences est le produit de la seconde. Il y a, par exemple, des principes de la théorie évolutionniste que les biologistes doivent respecter. Mais de tels principes sont des produits chèrement gagnés par l'expérience acquise lors de l'application des modèles d'explication darwiniens à un monde exhibant autant de facteurs complexes que la "dérive génétique " (genetic drift) ou l'instabilité environnementale. Ces principes ne découlent pas d'une réflexion sur des problèmes épistémologiques généraux.

Il me faut toutefoir préciser que la position à laquelle je souscris n'est pas, et il s'en faudrait de beaucoup, une version de l'anarchisme feyerabendien; une position que Robert, avec raison du reste. rejette tout spécialement. Pace Feyerabend, nous pouvons effectivement identifier une foule de choses qu'un bon scientifique ne devrait pas faire : ne pas violer le modus ponens, ne pas tirer d'inférences à partir d'un échantillon trop petit ou non représentatif, ne pas accepter des conclusions qui se fondent sur l'autorité de sources de crédit douteux, et ne pas manipuler leurs données outre mesure. Mais, pace Robert, je ne vois aucun véritable avantage à convertir un tel ensemble d'heuristiques négatives en une théorie épistémologique de la science. En effet, de telles heuristiques correspondent simplement aux aptitudes critiques élémentaires que se doivent de respecter tous les penseurs dans tous les aspects de leur vie. L'impossibilité d'une épistémologie adéquate de la science est, selon moi, un cas 
spécial de l'impossibilité d'une épistémologie générale adéquate. Ceci nous amène à un autre aspect essentiel du désaccord qui existe entre Robert et moi. Contester, comme je le fais, qu'il puisse y avoir une épistémologie univoque de la science, implique immédiatement qu'il ne peut pas exister de critères épistémologiques de démarcation entre science et non-science. Je soutiens que la science est tout simplement une disposition assez inhabituelle à appliquer de manière consciente, et prudemment contrōlée, la batterie d'aptitudes de raisonnement dont nous avons été dotés par l'évolution. La prudence et la mise sur pied de dispositifs institutionnels de contröle sont rendues nécessaires par le fait que les scientifiques sont souvent amenés à déployer leurs capacités intellectuelies dans des domaines pour lesquels la sélection naturelle n'avait aucune raison de nous préparer, et dans lesquels, par conséquent, nous sommes maladroits. Naturellement, les poppériens, qui éprouvent un besoin idéologique d'avoir un critère de démarcation, mobjecteront que cette conception peut être aussi utilisée pour tendre la perche aux astrologues et aux créationnistes. À cela je répondrais que nous n'avons nullement besoin d'une méta-théorie de la science pour justifier la thèse que lastrologie et le créationnisme procèdent d'un mauvais raisonnement; en effet, leurs tenants violent constamment le modus ponens, infèrent à partir d'échantillons non représentatifs, etc. Si en affirmant que l'astrologie et le créationnisme ne sont pas scientifiques, nous voulons simplement dire qu'on ne devrait pas leur accorder de place à l'intérieur de nos institutions scientifiques, alors il est facile de voir que l'on peut soutenir cette thèse sans mème avoir besoin de s'appuyer à un soi-disant sens profond, philosophique, dans lequel ils ne seraient pas scientifiques.

Si la conception que j'ai soutenue venait un jour à ètre généralement acceptée, alors le livre de Robert viendrait à être vu comme une évaluation bien pesée et très bien informée d'une sous-discipline morte : la philosophie générale de la science. Mais cela ne constituerait pas pour autant le glas de la philosophie de la science en général. Nous continuerions à attendre des philosophes de la physique qu'ils s'occupent des problemes conceptuels qui peuvent émerger de la physique, des philosophes de la biologie qu'ils se chargent des problemes conceptuels qui émergent de la théorie biologique et ainsi de suite. Ce me semble être une affirmation sociologique vraie que de dire que les tendances sont, en philosophie des sciences, en train de se developper dans le sens que je favorise. Naturellement, il n'est pas exclu qu'il apparaisse dans quelques années que j'aie suivi une mode intellectuelle éphémère pendant que des philosophes comme Robert auraient sagement gardé le bon cap. Je ne crois pas que cela se produise un jour, mais si jamais il devait en ètre de même, alors Les mécanismes de la découverte scientifique mériteront plusieurs ré-éditions.

Don Ross

Département de philosophie

Université d'Ottawa 